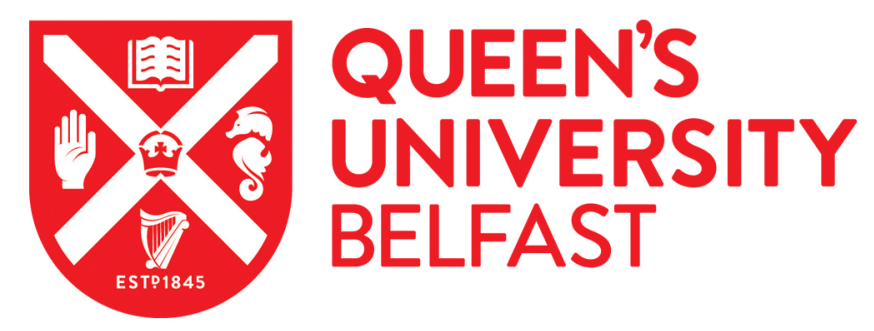

\title{
Analysing the Performance of a Real-Time Healthcare 4.0 System using Shared Frailty Time to Event Models
}

Marshall, A. H., \& Novakovic, A. (2019). Analysing the Performance of a Real-Time Healthcare 4.0 System using Shared Frailty Time to Event Models. In 2019 IEEE 32nd International Symposium on Computer-Based Medical Systems (CBMS) (pp. 622-627). ( 2019 IEEE 32nd International Symposium on Computer-Based Medical Systems (CBMS)). Institute of Electrical and Electronics Engineers Inc.. https://doi.org/10.1109/CBMS.2019.00129

Published in:

2019 IEEE 32nd International Symposium on Computer-Based Medical Systems (CBMS)

Document Version:

Peer reviewed version

Queen's University Belfast - Research Portal:

Link to publication record in Queen's University Belfast Research Portal

Publisher rights

Copyright 2020 IEEE. This work is made available online in accordance with the publisher's policies. Please refer to any applicable terms of use of the publisher.

\section{General rights}

Copyright for the publications made accessible via the Queen's University Belfast Research Portal is retained by the author(s) and / or other copyright owners and it is a condition of accessing these publications that users recognise and abide by the legal requirements associated with these rights.

Take down policy

The Research Portal is Queen's institutional repository that provides access to Queen's research output. Every effort has been made to ensure that content in the Research Portal does not infringe any person's rights, or applicable UK laws. If you discover content in the Research Portal that you believe breaches copyright or violates any law, please contact openaccess@qub.ac.uk. 


\title{
Analysing the performance of a real-time Healthcare 4.0 system using shared frailty time to event models
}

\author{
Adele H Marshall ${ }^{*}, 1$ and Aleksandar Novakovic ${ }^{*}, 1$ \\ School of Mathematics and Physics \\ Queen's University Belfast, Northern Ireland, United Kingdom \\ *Email: \{a.h.marshall, a.novakovic\}@qub.ac.uk \\ ${ }^{1}$ Sharing first authorship
}

\begin{abstract}
This paper introduces the real-time Healthcare 4.0 system, the VILIAlert system and a new approach that we propose for the robust assessment of it's performance. The VILIAlert system alerts clinicians when a patient's tidal volume value rises above the clinically accepted level of $8 \mathrm{ml} / \mathrm{kg}$ as beyond this point $(>8 \mathrm{ml} / \mathrm{kg})$, a patient is considered high risk of permanent damage to their lungs. In order to ensure success with the VILIAlert system, the ideal scenario is to ensure that as soon as patients in the Intensive Care Unit experience tidal volume values beyond the $8 \mathrm{ml} / \mathrm{kg}$ level, a clinical intervention can be carried out so to minimise the risk of patients ever having permanent damage. The approach has been implemented in the Intensive Care Unit at the Royal Victoria Hospital Belfast, Northern Ireland demonstrating the potential for such an approach to be used across all hospitals in the region.
\end{abstract}

Keywords: Healthcare 4.0, real time alerts, performance, frailty models.

\section{INTRODUCTION}

Patients admitted to an intensive care unit (ICU) are in a critical condition requiring their vital signs to be constantly tracked so that appropriate treatment can be administered in a timely manner. The four main routinely monitored vital signs are blood pressure, body temperature, respiration rate and pulse rate which indicate the state of a patient's essential body functions. Fluctuations in these vital signs may indicate the changes in functioning of the patients' body, the severity of their illness and the urgency in which it needs to be treated [1]. The body of an ICU patient is very frail. They usually cannot sustain themselves, and hence they need to be attached to various organ support instruments which have a purpose to keep them alive until their condition becomes less urgent.

One of the most commonly used instruments for providing organ support in the ICU wards are mechanical ventilators which are used to provide breathing assistance to patients who have developed a respiratory failure. However, growing evidence from preclinical and clinical trials involving ventilated animal and human subjects respectively, shows that the mechanical ventilation is a harmful intrusive intervention which if not performed properly has a strong tendency to permanently damage the lungs [2]. This damage dealt to the lungs by injurious ventilation is also known as the ventilator induced lung injury (VILI), with even the healthy lungs not immune [3]. By adopting lung protective ventilation (LPV) strategies in which the patients are ventilated with the tidal volumes $<8 \mathrm{ml} / \mathrm{kg}$ of ideal body weight (IBW), the harmful effects of ventilation are preventable [4] [5] [6].

The majority of these instruments used for the vital parameters monitoring and organ support are designed such that they can emit the patients' readings in compliance with the set of Health Level 7 (HL7) standards. This set of standards defines the framework for exchange, sharing and retrieval of electronic health information, which are necessary for smooth integration between a variety of systems. This data is normally stored in electronic medical records (EMR) for the purpose of further processing [1] [7]. The continuous flow of data from multiple different sources creates a highly complex dynamic environment thus raising the need for an appropriate real-time clinical response. However, this may also potentially lead to information and cognitive overload for clinicians and healthcare providers who are attempting to make sense of the high volume of data. As a result slow and inconsistent responses to incoming data can occur [8] and have a negative impact to patient outcomes such as reduced survival chances, or longer organ support requirements.

For example, despite the numerous studies demonstrating the benefits of LPV, current evidence shows that less than $50 \%$ of all ventilated patients receive it in clinical settings [9] [10] [11]. To facilitate the proper use of LPV, we have developed the real-time smart alerting, and Healthcare 4.0 compliant, clinical decision support (CDS) system called VILIAlert. It was deployed at the Royal Victoria Hospital, Belfast, with the goal to continuously monitor patient tidal volume (TVs) against a set of thresholds and provide the appropriate real time alerts when the system predicts that the configured ventilatory settings may lead to VILI. As it was operating in the Regional ICU which can admit up to a maximum of 20 patients, previous work investigated its scalability potential to adopt the system on a hospital wide level, and got very promising results [12].

There are very few studies focused on using CDS to improve quality of mechanical ventilation, and to our knowledge no studies that have investigated the performance of real-time driven smart alerting clinical systems. Once the alert has been generated, it is a particularly challenging task to distinguish genuine from clinically insignificant alerts without clinical input, which may lead to alert fatigue [13]. Because of the unlabelled data, standard classification or regression performance evaluation metrics such as area under the curve 
(AUC), $\mathrm{R}^{2}$ or root mean square error (RMSE) become unsuitable for the task, leading to the need for the development of new performance estimation techniques. Using the data collected during the period of operation of the VILIAlert system, we propose a novel approach inspired by survival analysis techniques which can be used for analysing the performance of the real-time Healthcare 4.0 systems. The paper is organized as follows.

In Section II we describe the architecture of the VILIAlert system and set up the theoretical foundations for using survival analytics in the performance evaluation of unlabelled real-time datasets. In the Section III, we present the results obtained by applying techniques to collect the VILIAlert data set. In Section IV we provide a discussion of the results and the advantages and disadvantages of introducing survival analytics based evaluation techniques, as well as provide the closing remarks in which we discuss the plans of our future research.

\section{METHODS}

\section{A. An Architectural Overview of the VILIAlert System}

Mechanical ventilators, like other organ support and vital signs tracking instruments produce data compliant with the HL7 protocol. Using TCP at the transport layer, emitted patients' data are commonly transferred via an IP network to an Electronic Medical Record (EMR) for the storing and processing purposes. VILIAlert is designed to run alongside the EMR and to continuously monitor the ventilation process. The VILIAlert system consists of two components (Fig. 1):

- Database - used for storing routinely collected anonymized patient data: patient IDs, gender, height, and TV readings received from the ventilators at minute time intervals. In order to be able to perform post-hoc analysis of the system's performance we also store the information describing the time of alert generation, and corresponding anonymized patient details.

- Analytics Kernel-designed to continuously monitor patients' TVs and generate an alert if the set of threshold metrics is violated. Averaged TV values over 15 minute intervals are calculated $\left(\mathrm{TV}_{15}\right)$, and if these averaged values exceed $8 \mathrm{ml} / \mathrm{kg}$ for four consecutive intervals, the analytics kernel triggers the alert and notifies relevant clinicians and healthcare providers via SMS. To prevent alert fatigue, the analytics kernel is configured so that if an alert is generated for some patient $I$, the kernel enters into so called "sleep mode" and stops generating alerts for this particular patient in the period of the next 12 hours until the alert has been dealt with.

VILIAlert was deployed to the Royal Victoria Hospital, Belfast in November 2015 and continuously monitored patients' ventilation process for almost two years. As we started gaining operational experience, in the first 10 months since activation, the entire system went through several tuning stages in which we refined the data collection and alerting processes. This paper reports on the data collected between 31.08.2016 and 26.10.2017, the dates when the system entered a stable state and stopped being in use, respectively.

\section{B. Survival Analysis Approach for Performance Estimation}

Although VILIAlert stored the timestamps of generated alerts, clinicians and healthcare providers provided no feedback on how genuine the alerts actually were. The resulting unlabelled dataset prevented the application of commonly used metrics for analyzing systems' performance in the classification and regression analysis, for evaluating VILIAlerts' accuracy. Instead this intelligence had to be built into the system which had to be able to distinguish the genuine alerts from those that were false.

To investigate whether the generated alerts are genuine or not, we focused our analysis on the period of time covering the first six hours after their generation. We named this timewindow as APA (Analysed Post Alert Time-Window) and segregated it into 24 consecutive blocks, each representing one 15 minute interval. Because the alert is triggered if $\left(\mathrm{TV}_{15}\right.$ $>8 \mathrm{ml} / \mathrm{kg}$ for four consecutive 15 minute intervals (i.e. 4 consecutive blocks), we expected $\mathrm{TV}_{15}$ to remain above 8 $\mathrm{ml} / \mathrm{kg}$ throughout all 24 blocks of APA. Each block complying with these expectations was denoted as a "nondefective block" (NDB), otherwise it was marked as "defective block" (DB). In the perfect scenario, an APA corresponding to a genuine alert should consist of NDBs only, however this may not always be the case because of the fact that the six hours is a long period of time during which many external factors may affect mechanical ventilation (e.g. clinical intervention, changing between control and support modes of mechanical ventilation, etc.) which will be reflected

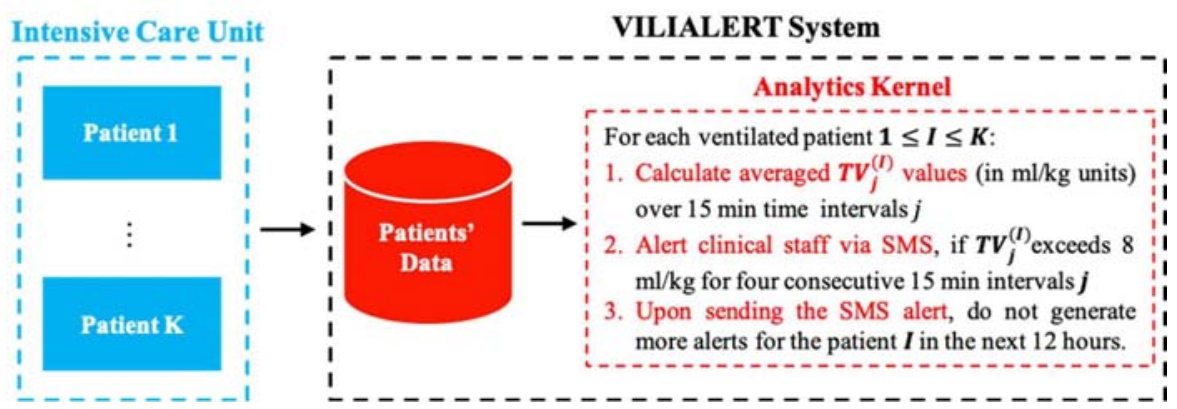

Figure 1. An architectural overview of the VILIAlert System 
in lower $\mathrm{TV}_{15}$ values and therefore result in the appearance of DBs during APA.

By marking APA blocks as DB or NDB, we transformed a set of continuous $\mathrm{TV}_{15}$ readings into a set of binary data, enabling us to approach VILIAlert's performance assessment using survival analysis. Survival analysis is a collection of methods used to model the time until an event of interest occurs (survival time). Traditionally, as the name suggests, the event of interest was often the patient recovering from a disease hence the time until this happens was known as survival time. Typical to survival analysis is the highly skewed nature of the survival times when plotted on a graph which generally will peak at the beginning and slowly tail off to the right as fewer patients will experience the event as time progresses. A common feature in survival analysis is that of censoring where the patient may not have experienced the event over the course of the study period. Survival analysis provides the ability of still being able to use the information regarding such a patient until the point where no further information is recorded [14].

In terms of the VILAlert clinical decisions support system proposed in this paper, the DB is the desired event that the occurs in the study, and our goal is to predict the survival time at which point (i.e. at which APA block position ) the $\mathrm{TV}_{15}$ metric is going to drop below $8 \mathrm{ml} / \mathrm{kg}$ upon, that is, the alert generation. Therefore, the time to the event occurrence is defined by the position of the first APA block which is marked as the DB, with the longer times to event indicating better performance of the alerting algorithm. On the other hand, genuine alerts having all APA blocks marked as NDBs are said to be censored.

This can be best illustrated with Fig. 2 where each row represents one APA segregated into 24 blocks (i.e. 15 minute intervals). Each block can be denoted with either 0 or 1 representing to $\mathrm{NDB}$ or $\mathrm{DB}$, respectively. It is worth highlighting the third APA as it belongs to a genuine alert as all the blocks are marked as NDB. In survival analysis terms, this represents a censored event. On the other hand the first and second APAs, have the time to event occurrence of 1 (15 minutes) and 18 (270 minutes) respectively.

The initial analysis in this study considers the commonly used Kaplan-Meier (KM) method to estimate the overall survival function from the raw VILIAlert data. This allows for an the investigation of the probabilities of the first DB block in APA, appearing at some point of time $t=i \times 15 \mathrm{~min}$, where $1 \leq i \leq 24 \mathrm{i}$ the position of the block in APA (i.e. the probability of having $\mathrm{TV}_{15}>8 \mathrm{ml} / \mathrm{kg}$ up until the timestamp $t$ ). The KM methods also allows for the consideration of other influencing factors (known as covariates) and their influence on the estimated survival curves. Hence, the same KM approach is used to investigate the VILIAlert's performance with respect to the covariate gender to consider each gender separately, by comparing their survival distributions. For testing statistical significance of the difference in survival distributions, the log-rank test is used. This information allows for the understanding of whether the patient's gender is associated with increased risk of receiving injurious ventilation associated with VILI.
The Cox proportional hazard $(\mathrm{CPH})$ model is a well known survival method used to model the time to occurrence

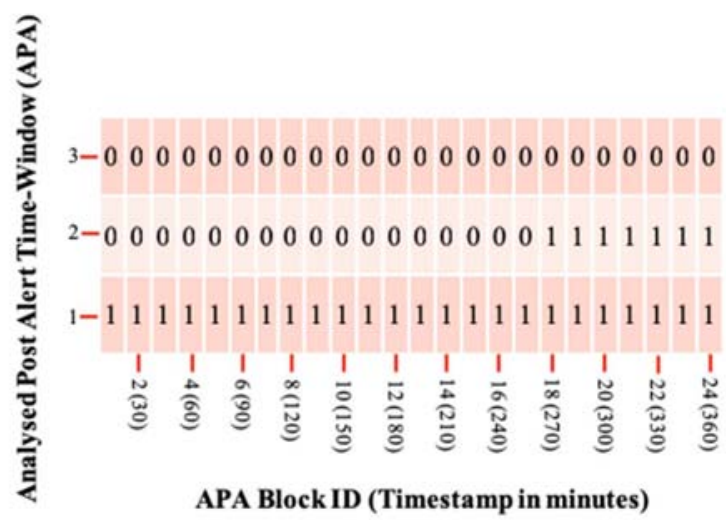

Figure 2. A schematic overview of three analysed post alert timewindows (APA) covering the period of 6 hours upon the alert generation. Defective and non-defective blocks are denoted with the numbers 1 and 0 respectively.

of the first DB in APA (the survival time). An assumption of this approach, is the need for proportional hazards hence the following accelerated failure time (AFT) models: Weibull, exponential, logistic, lognormal and log-logistic models are also applied to investigate the best fit model for the time until the first DB if such a case were to arise that the hazards are non proportional. The most frequent approach to applying these models in practice is by assuming homogeneity of data in which the subjects are independent of each other, or in our case assuming alert independence regardless of the patient who generated it. However, having in mind the fact that each patient can generate one or more alerts, the analysis of the VILIAlert data has also considered their lognormal and gamma shared frailty extensions on patient level to take account of this. More information about these models can be found in the literature on survival analysis and their shared frailty extensions [15] [16]

In total 54 models (univariate or multivariate in nature) were constructed for the VILIAlert data, each using the different combinations of covariates and different survival types. The patient's gender and height were considered the candidate covariates in standard models, while in frailty models patient IDs (the variable PID) were also used as the shared frailty term.

The performance of the resulting survival models were assessed using the Akaike Inference Criterion (AIC), a goodness of fit metric commonly used for comparing performance of models in survival analysis and other machine learning techniques. It is based on the concept of entropy, penalising the models with large number of parameters which hence can be seen as a trade-off between bias and variance (or in other words, a balance between accuracy and complexity) with the lower scores indicating better performance [17]. AIC can be calculated as follows:

$$
A I C=-2 \times(\log (L)-P)
$$


where $L$ and $P$ denote the model maximum likelihood value, and the number of parameters in the model, respectively. If two models have similar AIC scores, model selection is performed using the log-likelihood ratio test and the best model to be chosen as the one with the fewer number of parameters.

Survival analytics was performed using the R v3.5.0 programming language [18]. Survival models were implemented using R's survival library [19] [20]. A tutorial on implementing lognormal and gamma shared frailty models using the survival package can be found in the paper by Austin [14].

\section{RESULTS}

During the period of operation of the VILIAlert system, 560 patients generated 1450 alerts in total. Of that number 298 were male patients who were involved in generating 691 alerts. Thus suggesting that even though there were more male patients who were generating alerts, the number of alerts being generated was greater for female patients who had more alerts generated relative to the number of patients.

Given the highly skewed nature of the times to first DB, the median overall time is used to report time to the first DB block appearing in the APA (i.e. the median of overall survival time). This is 180 minutes. When the overall survival time is stratified by gender, the median survival times for males and females were 105 and 300 minutes respectively. At 95\% confidence level, the log-rank test indicates that there is a very highly significant difference in survival distributions between the genders $\left(\chi_{1}^{2}=65.8, p<0.0001\right)$.

TABLE I. PERFORMANCE COMPARISON OF THE TOP 5 SURVIVAL MODELS

TABLE II.

\begin{tabular}{|c|c|c|c|c|}
\hline ID & $\begin{array}{c}\text { Survival } \\
\text { Method }\end{array}$ & Covariates & AIC & Loglik. \\
\hline 1 & $\begin{array}{c}\text { Frailty: } \\
\text { Lognormal }\end{array}$ & $\begin{array}{c}\text { Gender, } \\
\text { height, } \\
\text { lognorm.frailty(PID) }\end{array}$ & 6693.48 & -3062.06 \\
\hline 2 & $\begin{array}{c}\text { Frailty: } \\
\text { Lognormal }\end{array}$ & $\begin{array}{c}\text { height, } \\
\text { lognorm.frailty(PID) })^{1}\end{array}$ & 6693.75 & -3062.83 \\
\hline 3 & $\begin{array}{c}\text { Frailty: } \\
\text { Loglogistic }\end{array}$ & $\begin{array}{c}\text { Gender, } \\
\text { height, } \\
\text { lognorm.frailty(PID) }\end{array}$ & 6698.49 & -3024.51 \\
\hline 4 & $\begin{array}{c}\text { Frailty: } \\
\text { Loglogistic }\end{array}$ & $\begin{array}{c}\text { height, } \\
\text { lognorm.frailty(PID) })^{1}\end{array}$ & 6698.88 & -3025.15 \\
\hline 5 & $\begin{array}{c}\text { Frailty: } \\
\text { Loglogistic }\end{array}$ & $\begin{array}{c}\text { Gender, } \\
\text { height, } \\
\text { gamma.frailty(PID) }\end{array}$ & 6713.15 & -2985.05 \\
\hline & 1. Lognormal shared frailty on patient level; 2. Gamma shared frailty on patient level \\
\hline
\end{tabular}

The corresponding KM estimates are provided in fig. 3 . Fig. 3. a) illustrates the estimated survival curve for the VILIAlert data for time until first DB for each patient. This is typical of the survival curve shape where it is highly skewed in nature with the majority of patients experiencing the event quickly but some taking longer to experience the event hence featuring in the tails of the distribution. Fig. 3. b) interestingly highlights the difference between male and female patient survival where the first DB blocks in the APAs of the males are occurring much sooner in their stay in ICU than the female patients. If we compare the quality of generated alerts between the genders, this would indicate better VILIAlert performance in the female compared to the male patients because of the higher survival probabilities. However, with almost three times longer median survival time, this unfortunately also suggests poorer ventilatory treatments in female patients compared to the males, as they are ventilated with the injuriously high TVs $(>8 \mathrm{ml} / \mathrm{kg})$ for a much longer period of time, exposing them to the higher risk of developing VILI.

TABLE III. A SUMMARY OF THE BEST PERFORMING MODEL

\begin{tabular}{|c|c|c|}
\hline Frailty: Lognormal & Beta Coefficient & p-value \\
\hline Intercept & 12.2310 & $<0.0001$ \\
\hline height & -0.0598 & $<0.0001$ \\
\hline lognorm.frailty(PID) ${ }^{1,2}$ & \multicolumn{2}{|c|}{$<0.0001$} \\
\hline \multicolumn{2}{|c|}{ 1. Lognormal shared frailty on patient level; 2 . Variance of the frailty distr. $\theta=0.901$}
\end{tabular}

a)

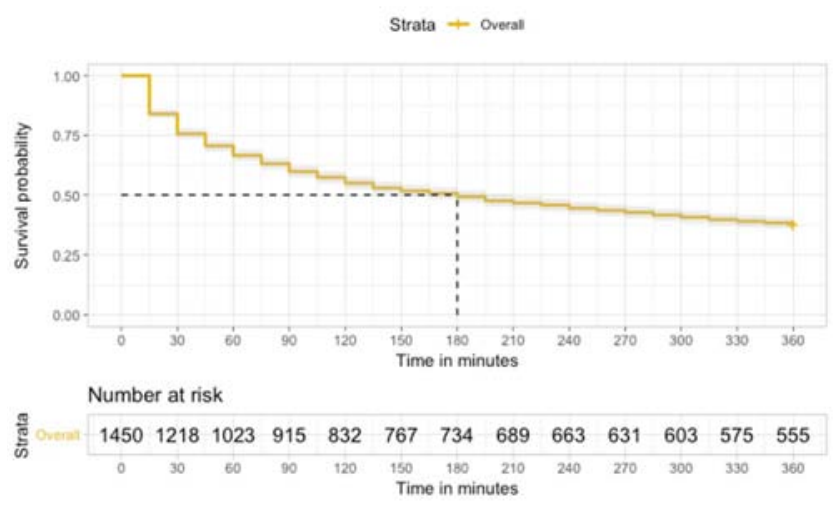

b)

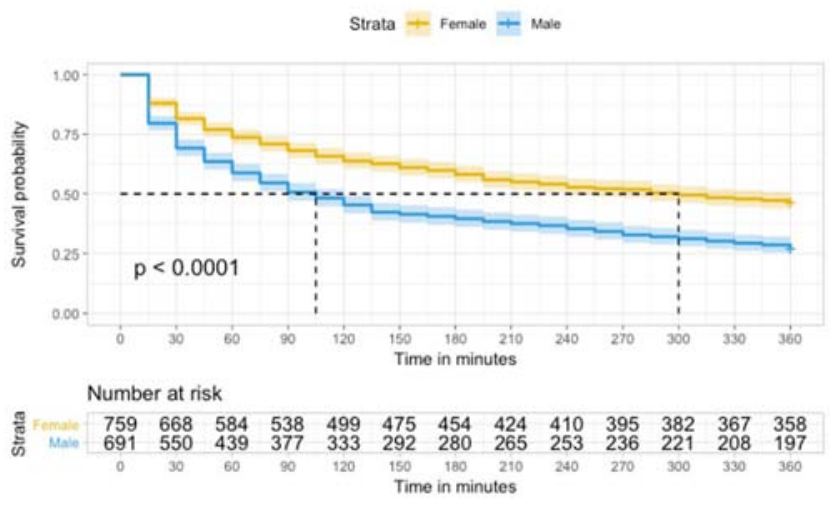

Figure 3. Kaplan-Meier estimates of: a) overall survival probability b) survival probabilities stratified by gender. Dashed lines indicate the median survival times.

Out of the 54 models created to represent the survival data, the AIC criteria selects the top 5 to be frailty AFT models. The AIC and log-likelihood scores are presented in Table 1. The top two performing models were lognormal shared frailty models with the lognormal baseline function. The loglikelihood ratio test indicates that there is no significant 
difference between the first two top performing models $\left(\chi_{1}^{2}=\right.$ $1.54, p=0.215$ ), and therefore the optimal model is the one with the smaller number of covariates, i.e. the model with ID $=2$. Hence the best model has patient height but not gender.

At the $95 \%$ significance level, the p-value suggests a very strong association between the patients' height and survival time. Keeping in mind that the patients' height is measured in centimetres, this suggest that with each centimetre of increase in height, the survival probability is reduced by a factor of 0.058 therefore implying that the taller the patient is, the lower the risk of receiving injurious ventilation (shorter patients have poorer treatments). The variance of the frailty distribution $\theta=0.901(p<0.0001)$ suggests a great degree of heterogeneity present in the APA dataset.

\section{DISCUSSION}

In this paper, we introduce a real time Healthcare 4.0 system which has been applied to an Intensive Care Unit (ICU) for critically ill patients. The VILIAlert system alerts clinicians when a patient's tidal volume value rises above the clinically accepted level of $8 \mathrm{ml} / \mathrm{kg}$ as beyond this point ( $>8 \mathrm{ml} / \mathrm{kg}$ ), a patient is considered high risk of permanent damage to their lungs. In order to ensure success with the VILIAlert system, the ideal scenario is to ensure that as soon as patients go beyond the $8 \mathrm{ml} / \mathrm{kg}$ level, that a clinical intervention is carried out so to minimise the risk of patients ever having permanent damage. Hence the motivation for this paper, to model the time until such events occur using survival analysis methods. The resulting best model is the frailty model which includes the height as a covariate. This is consistent with previous literature which suggests that the ventilators are less effective for female patients who in general are shorter in height. In particular, previous findings have shown that the female patients and those with the height of $<165 \mathrm{~cm}$ are at an increased risk of receiving injurious ventilation [21]

The approach introduced in this paper, for measuring the performance based on the survival analysis, could be used for the development of other new algorithms and their crosscomparison. We provided the framework (guidelines) applicable not just to our use case but to any real-time system capturing non-labelled data. In fact, it can be used for understanding systems behaviour as well as for the development of new algorithms and the cross comparison of their performance (something similar to the Kaplan-Meier Male vs Female curve which is shown in the fig 3. b)). Our aim is to use this approach on the existing dataset to develop more robust smart alerting algorithms.

One potential drawback of our approach is it does not fully take into account time dependent covariates which change over time and it takes as a failure time the appearance of the first DB block in APA. This is slightly too conservative an approach e.g. it is possible for APA to have only the $1^{\text {st }}$ block flagged as DB and have all the other blocks as NDB. This is an example of a genuine alert where the DB occurred probably due to the spurious ventilatory reading, but the algorithm will use time of death as 1 (i.e. $15 \mathrm{~min}$ since ventilation started) and ignore everything else. To overcome this problem, we propose survival models with time dependent covariates, the topic of our future research.

Also this raises a need for the development of new visualisation techniques which could help improve the understanding of the distribution of DB and NDB incidents throughout the APA.

\section{ACKNOWLEDGMENT}

The authors wish to acknowledge the Royal Victoria Hospital for facilitating the testing and validation of the VILIAlert system. We would also like to thank Dr Charles J Gillan (Institute of Electronics, Communications and Information Technology) and Dr Murali Shyamsundar (The Centre for Experimental Medicine, School of Medicine Dentistry and Biological Sciences) from Queen's University Belfast, for carrying out the conception and implementation of the VILIAlert system to gather the data.

\section{REFERENCES}

[1] K. Plathong and B. Surakratanasakul, "A study of integration Internet of Things with health level 7 protocol for real-time healthcare monitoring by using cloud computing," in The 2017 Biomedical Engineering International Conference (BMEiCON-2017), Hokkaido, Japan, 2017.

[2] A. S. Slutsky and V. M. Ranieri, "Ventilator-Induced Lung Injury," The New England Journal of Medicine, vol. 369, pp. 2126-2136, 2013.

[3] R. M. Determann and et al, "Ventilation with lower tidal volumes as compared with conventional tidal volumes for patients without acute lung injury: a preventive randomized controlled trial," Critical Care, vol. 14, no. 1, p. R1, 2010.

[4] M. B. P. Amato and et al, "Effect of a protective-ventilation strategy on mortality in the acute respiratory distress syndrome," The New England Journal of Medicine, vol. 338, pp. 347-354, 1998.

[5] C. Putensen, N. Theuerkauf, J. Zinserling, H. Wrigge and P. Pelosi, "Meta-analysis: ventilation strategies and outcomes of the acute respiratory distress syndrome and acute lung injury," Annals of Internal Medicine, vol. 151, no. 8, pp. 566576, 2009.

[6] A. Serpa Neto, "Lung-protective ventilation in intensive care unit and operation room: Tidal volume size, level of positive end-expiratory pressure and driving pressure ( $\mathrm{PhD}$ Thesis)," Faculty of Medicine, University of Amsterdam, 2017.

[7] Health Level Seven International, "About HL7," [Online]. Available: https://bit.ly/2tkavDk. [Accessed 5 February 2019].

[8] M. Ghassemi, L. A. Celi and D. J. Stone, "State of the art review: the data revolution in critical care," Critical Care, vol. 19 , no. 1, p. $118,2015$. 
[9] A. J. Walkey and R. S. Wiener, "Risk Factors for Underuse of Lung Protective Ventilation in Acute Lung Injury," Critical Care, vol. 27, no. 3, pp. 323.e1-323.e9, 2012.

[10] W. Checkley, R. Brower, A. Korpak and B. T. Thompson, "Effects of a clinical trial on mechanical ventilation practices in patients with acute lung injury," American Journal of Respiratory and Critical Care Medicine, vol. 177, no. 11, pp. 1215-1222, 2008.

[11] R. Kalhan and et al., "Underuse of lung protective ventilation: analysis of potential factors to explain physician behavior.," Critical Care Medicine, vol. 34, no. 2, pp. 300-306, 2006.

[12] C. J. Gillan, A. Novakovic, A. H. Marshall, M. Shyamsundar and D. S. Nikolopoulos, "Expediting assessments of database performance for streams of respiratory parameters," Computers in Biology and Medicine, vol. 100, pp. 186-195, 2018.

[13] V. Herasevich and et al., "Limiting ventilator-induced lung injury through individual electronic medical record surveillance," Critical Care Medicine, vol. 39, no. 1, pp. 3439, 2011.

[14] P. C. Austin, "A Tutorial on Multilevel Survival Analysis: Methods, Models and Applications," International Statistical Review, vol. 85, no. 2, pp. 185-203, 2017.

[15] M. Mills, Introducing Survival Analysis and Event History Analysis, Thousand Oaks, CA: SAGE, 2011.

[16] A. Wienke, Frailty Models in Survival Analysis, New York: Chapman and Hall/CRC, 2010.

[17] S. J. Wang, J. Kalpathy-Cramer, J. S. Kim, C. D. Fuller and C. R. Thomas, "Parametric survival models for predicting the benefit of adjuvant chemoradiotherapy in gallbladder cancer," in AMIA Annual Symposium Proceedings, 2010.

[18] R Core Team, "R: A language and environment for statistical computing," R Foundation for Statistical Computing, Vienna, Austria, 2018.

[19] T. M. Therneau, "A Package for Survival Analysis in S," 2015. [Online]. Available: https://CRAN.Rproject.org/package $=$ survival.

[20] T. M. Thernau and P. M. Grambsch, Modeling Survival Data: Extending the Cox Model, New York: Springer, 2000.

[21] A. F. Bustamante, C. L. Wood, Z. V. Tran and P. Moine, "Intraoperative ventilation: incidence and risk factors for receiving large tidal volumes during general anesthesia," BMC Anesthesiology, vol. 11, p. 22, 2011. 